\title{
SCALABLE OPTICAL TRACKING A Practical Low-cost Solution for Large Virtual Environments
}

\author{
Steven Maesen and Philippe Bekaert \\ Hasselt University - $t U L$ - IBBT, Expertise Centre for Digital Media, Wetenschapspark 2, 3590 Diepenbeek, Belgium \\ $\{$ steven.maesen, philippe.bekaert $\} @$ uhasselt.be
}

\begin{abstract}
Keywords: Optical tracking, Wide-area, Low-cost, Scalable.
Abstract: Navigation in large virtual reality applications is often done by unnatural input devices like keyboard, mouse, gamepad and similar devices. A more natural approach would be letting the user walk through the virtual world as if it was a physical place. This involves tracking the position and orientation of the participant over a large area. We propose a pure optical tracking system that only uses off-the-shelf components like cameras and LED ropes. The construction of the scene doesn't require any off-line calibration or difficult positioning, which makes it easy to build and indefinitely scalable in both size and users.

The proposed algorithms have been implemented and tested in a virtual and a room-sized lab set-up. The first results from our tracker are promising and can compete with many (expensive) commercial trackers.
\end{abstract}

\section{INTRODUCTION}

A big step towards the immersive feeling in virtual reality is the ability to walk through the virtual environment instead of pushing buttons to move. This requires a wide-area tracking system. But many commercial systems (acoustic, mechanic, magnetic,...) don't support this kind of scalability.

An example of such a system is the optical tracking system HiBall (Welch et al., 2001), which provides great speed and accuracy. The HiBall tracker uses a special-purpose optical sensor and active infrared LEDs. Their use of specially designed hardware probably explains why the system is so expensive.

Our main goal is to build a pure optical wide-area tracking system at a low cost using only off-the-shelf components. We also don't expect the position of each LED to be known or calibrated, which makes the construction of the set-up fast and easy. By using only passive markers, we can support an indefinite number of cameras to be tracked because there is no synchronization required between them and each camera is a self-tracker (Bishop, 1984). It also makes it very easy to expand the working volume indefinitely provided you have sufficient ceiling space.

\section{RELATED WORK}

Tracking of participating persons has been a funda- mental problem in virtual immersive reality from the very beginning (Sutherland, 1968). In most cases, special-purpose hardware trackers were developed with usually a small (accurate) working area. Most trackers are used to track the head of a person wearing a head mounted display (HMD) to generate the virtual world from their point of view. Many different technologies have been used to track HMDs: mechanical, magnetic, acoustic, inertial, optical, ... and different kinds of hybrid combinations.

The first HMD by Ivan Sutherland (Sutherland, 1968) used a mechanical linkage to measure the head position. Mechanical trackers are very fast and accurate, but suffer from a limited range because the user is physically attached to a fixed point.

Magnetic-based systems on the other hand don't have a physical linkage with the magnetic source, in fact they don't even need a line-of-sight between its source and receiver. But they suffer from a limited range (as do all source-receiver systems that use only 1 source) and are not very scalable. Also metal or other electromagnetic fields cause distortions in the pose measurements.

Acoustic tracking systems use ultrasonic sounds to triangulate its position. This system does require a line-of-sight between source and receiver and also suffers from a limited range. The accuracy of the system also depends on the ambient air conditions.

Inertial tracking systems use inertia to sense position and orientation changes by measuring accel- 
eration and torque. This type of system doesn't require any source or markings in the environment and therefore has an unlimited range. However this also means that there is no absolute position given and the measured position quickly drifts from the exact position. Inertial self-trackers are often combined with vision systems (optical trackers) to counteract the weak points of each other. An example of such a hybrid tracker is the VIS-Tracker (Foxlin and Naimark, 2003; Wormell et al., 2007). The VIS-Tracker uses paper patterns for absolute reference to counter drift from its inertial tracker. Filming paper markings has the disadvantage of needing enough light and a long shutter time, which increases the effect of motion blur. The patterns also need to be calibrated before use.

With the rapid rise of CPU speeds and advances in affordable camera systems, computer vision based tracking systems can operate in real-time. But the best vision based systems, like the HiBall tracker (Welch et al., 2001), still are very expensive and require special hardware. Optical systems need line-of-sight to detect feature points in the world, but can have a great accuracy and update rate with hardly any latency as shown by the commercially available HiBall tracker.

Another low-cost optical tracking system was developed at MERL (Raskar et al., 2007) which estimates position, orientation and incident illumination at $124 \mathrm{~Hz}$. They use cheap electronic components to build a projector of light patterns. The receiver uses these coded light signals to estimate position and orientation. But the system only has a limited working area of a few meters.

More information about all these techniques can be found in the course "Tracking: Beyond 15 Minutes of Thought" by Gary Bishop, Greg Welch and B. Danette Allen (Allen et al., 2001).

Our goal is to make a wide-area tracking system that allows the user to walk around in a building. Most systems discussed above only have a limited range and therefore aren't really suited for this task. The VIS-Tracker and the HiBall tracker are designed for the same goal. But the VIS-Tracker mostly relies on its inertial tracker and uses its camera secondary for recalibration of its absolute position. The HiBall on the other hand is also a pure optical tracking system. In fact their set-up shows similarities in the way that we also chose a inside-looking-out system with markers on the ceiling.

The HiBall system uses a specially designed sensor with multiple photo diodes that measures the position of each sequentially flashed infra-red LED in the specially designed ceiling. The system uses the 2D-3D correspondences of each LED to accu- rately estimate the position and orientation of the HiBall as discussed by Wang (Wang et al., 1990) and Ward (Ward et al., 1992). The final version of the HiBall tracker uses a single-constraint-at-a-time approach or SCAAT tracking (Welch, 1997).

We take a different approach to estimate rotation and position. We calculate the orientation from the vanishing points of the constructed lines parallel to the X- and Y-directions. This can be done separately from the cameras position. Camera calibration from vanishing points isn't a new technique. Caprile (Caprile and Torre, 1990) used vanishing points for off-line calibration of a stereo pair of cameras. Cipolla (Cipolla et al., 1999) used a similar technique to calibrate images of architectural scenes for reconstruction purposes.

\section{OVERVIEW TRACKING SYSTEM}

\subsection{Set-up of the Tracking System}

In our set-up, we have constructed a grid of LED ropes to identify the parallel lines in both the $\mathrm{X}$ and $\mathrm{Y}$ direction on the ceiling. We consider the distance between LED ropes to be known which is needed for position tracking.

The person or object that needs to be tracked will have a camera placed on top of it pointing upwards. We will consider the intrinsic parameters of the camera known. Those values are constant if we assume that the camera does not have a variable zoom or focus.

We choose to use LED ropes instead of ordinary markers because it makes the construction and detection in a real lab set-up easier. Choosing a LED rope saves a lot of time because we don't need to attach each LED separately and minimal extra wiring is required. Mass production of LED ropes also reduces production costs, which makes them relatively cheap. By using light sources instead of markers, we can decrease the shutter time of our cameras. This means we can have a higher camera frame rate, less background noise and motion blur in our images. This increases the performance and robustness of our tracking system.

Figure 1 shows our lab set-up tracking one person with virtual reality glasses by filming the grid of LED ropes on the ceiling. 


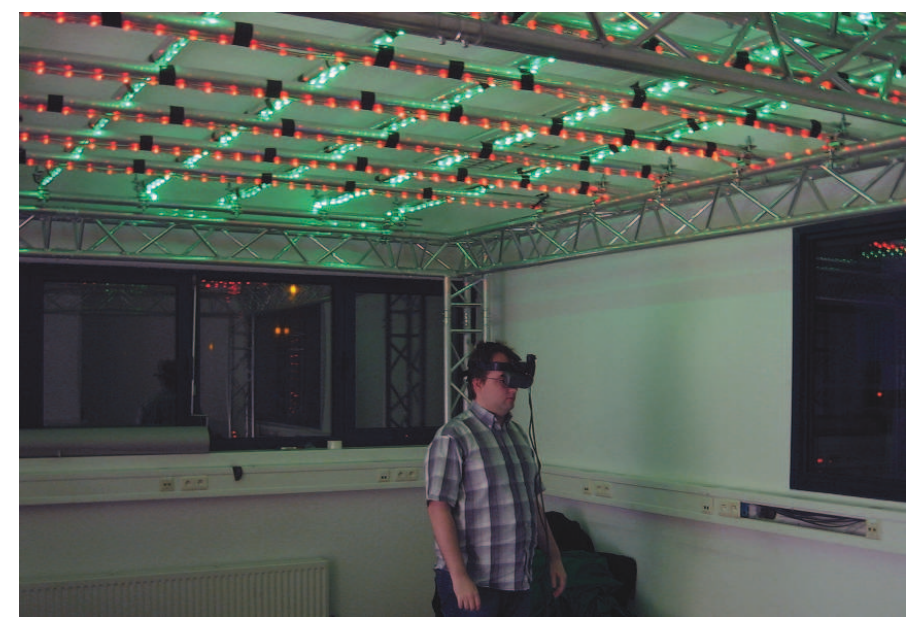

Figure 1: Our lab set-up with a 4 by 3 meter grid of LED ropes on the ceiling. The camera mounted on the virtual reality glasses tracks its position and orientation by filming this grid.

\subsection{Overview Algorithm}

Our tracking algorithm gets the images of the camera as input. The extrinsic parameters will be estimated in the following steps:

- Detection of the LED ropes

- Calculating orientation from vanishing points

- Calculating position with known orientation

These steps will be explained in detail in the following sections.

\section{DETECTION OF THE LED ROPES}

Our first task is the detection of our constructed grid in the input image. We first segment the individual LEDs by evaluating the hue value of each pixel. Then we use a simple 'flood fill' algorithm to cluster the pixels corresponding to a LED and retain only its center. That way we speed up the line detection considerably and eliminate a lot of random noise.

Secondly if we use a real camera, we need to take lens distortion into account. The effects of lens distortion are clearly visible when using a lens with a wide field of view. It causes straight lines to bend, especially near the edges. This is something we want to avoid at all costs. Therefore we calculate the distortion parameters beforehand with 'GML Toolbox' (V.Vezhnevets and A.Velizhev, 2005) based on the image processing library OpenCV (Bradski, 2000). Unlike the undistortion function in OpenCV, we do not want to undistort entire images because that would be prohibitory slow. Instead we create a lookup table to undistort individual LEDs very fast.

Last step in the detection of the LED ropes is the line pattern recognition in the collection of detected LEDs. A mature technique for line pattern recognition is the patented Hough Transform (Hough, 1962).

\subsection{Hough Transformation}

In general the Hough transformation is a mapping of the input points to a curve in a dual parameter space. The parameterization of the pattern (in this case a line) determines the used parameter space and the shape of the dual curves. The most common used parameterization maps an input point $\left(x_{i}, y_{i}\right)$ to a sinusoidal curve in the $\rho \theta$-plane with equation:

$$
x_{i} \cos \theta+y_{i} \sin \theta=\rho
$$

The geometrical interpretation of the parameters $(\theta, \rho)$ is illustrated in figure 2 .
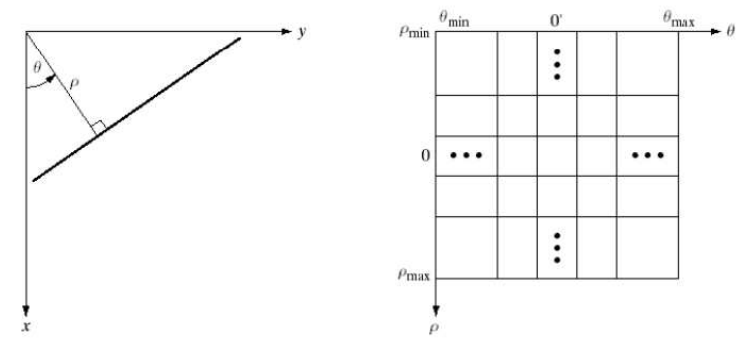

Figure 2: Left: Geometrical interpretation of the $(\theta, \rho)$ parameterization of lines. Right: Subdividing the parameter space into accumulator cells. (Image courtesy: (Gonzalez and Woods, 2001)).

The Hough algorithm (Gonzalez and Woods, $2001)$ attains its computational attractiveness $(O(N))$ 
by subdividing the parameter space into so-called accumulator cells (Figure 2). Each input point generates votes for every accumulator cell corresponding to its mapped sinusoid in parameter space. Finally the accumulator cells with the highest amount of votes (most intersections) represent the line patterns in the input space.

Although the Hough algorithm has a linear time complexity, it has a high constant cost of 'drawing' and searching for 'highlights'. Therefore most Hough transformations cannot be performed in real-time. Although we only have a small number of input points, the high constant cost weighed heavily on the trackers speed. Therefore we propose another line parameterization to speed up the calculations with a relatively small amount of input points.

\subsection{Line Parameterization with Circles}

To increase the speed and accuracy of the line detection, we propose to calculate the intersections in the Hough parameter space analytical. But this isn't trivial with sinusoidal curves (eq. 1). Therefore we propose a new parameterization that maps each input point $\left(x_{i}, y_{i}\right)$ to a circle in the $X Y$-plane with equation:

$$
\begin{aligned}
& \left(x-\frac{x_{i}+C_{x}}{2}\right)^{2}+\left(y-\frac{y_{i}+C_{y}}{2}\right)^{2} \\
& =\left(\frac{\sqrt{\left(x_{i}-C_{x}\right)^{2}+\left(y_{i}-C_{y}\right)^{2}}}{2}\right)^{2}
\end{aligned}
$$

with $\left(C_{x}, C_{y}\right)$ a fixed point in the image, like the principal point. This means that for every point $\left(x_{i}, y_{i}\right)$ we will construct a circle with the midpoint between $\left(x_{i}, y_{i}\right)$ and $\left(C_{x}, C_{y}\right)$ as center and radius equal to half the distance between these two points. The geometrical interpretation of this parameterization is shown in Figure 3.

The analytic intersection point of 2 circles is much easier to calculate than with 2 sinusoids. Given 2 circles with centers $C_{0}$ and $C_{1}$ and radiuses $r_{0}$ and $r_{1}$ and the fixed point $C$, we can calculate the second intersection point $P$ (first intersection point is $C$ ) as follows:

$$
P=\left(C_{0}+C_{1}\right)+\frac{r_{0}^{2}-r_{1}^{2}}{d^{2}}\left(C_{1}-C_{0}\right)-C
$$

with $d$ the distance between $C_{0}$ and $C_{1}$.

In practice, we will represent each line with this intersection point $P$. This 'line center' defines a line through this center $P$ and perpendicular to the direction $\overrightarrow{P C}$.

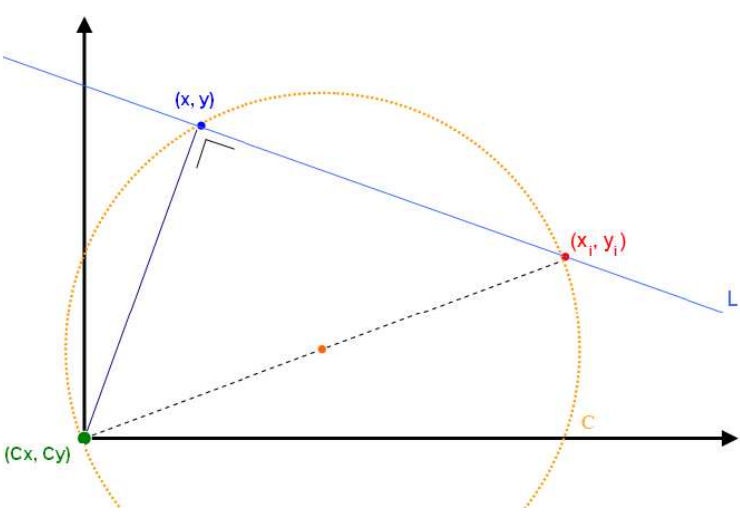

Figure 3: Geometrical interpretation of the circle parameterization of lines. Each line $L$ through input point $\left(x_{i}, y_{i}\right)$ is characterized by a point $(x, y) \in C$.

\subsection{Line Detection with the New Parameterization}

To detect lines in our input image, we try to estimate the most apparent line centers. For every LED in the input image, we try to estimate a local direction/line, e.g. by using the closest LED. Each of these lines defines a line center in the line parameterization space. Noise and measurement errors cause the local line centers representing the same line to slightly vary their position. This means that we must define a dynamic error bin around possible groups of line centers. The bins with the most points represent the most apparent line patterns in the image. The algorithm has an average complexity of $O\left(N^{2}\right)$. In applications with a small set of input points -like our tracker- our Hough algorithm greatly outperforms the standard algorithm.

\section{CALCULATING ORIENTATION FROM VANISHING POINTS}

The lines detected in the previous step are the projections of the constructed parallel lines. Therefore we know that each set of lines corresponding to one axis (one color of LEDs) intersects in a single point, the vanishing point. Classically the best fit intersection point of the lines is used as the vanishing point. This can introduce a lot of jitter in the vanishing point when the lines are nearly parallel. Therefore we propose to calculate the vanishing direction separate from the distance to the vanishing point. The vanishing direction is the direction from the principal point of the camera to the $2 \mathrm{D}$ position of the vanishing point. If we used the principal point as the fixed point in the line detection step $(\S 4.2)$, it can be shown 
that the vanishing direction $D$ equals the interpolation of two line directions $D_{1}$ and $D_{2}$ :

$$
D=\left\|l_{2}, p p\right\| * D_{1}+\left\|l_{1}, p p\right\| * D_{2}
$$

with $p p$ the principal point and $l_{1}, l_{2}$ the line centers from lines $L_{1}$ and $L_{2}$. Figure 4 gives a geometric representation of the interpolation. The vanishing

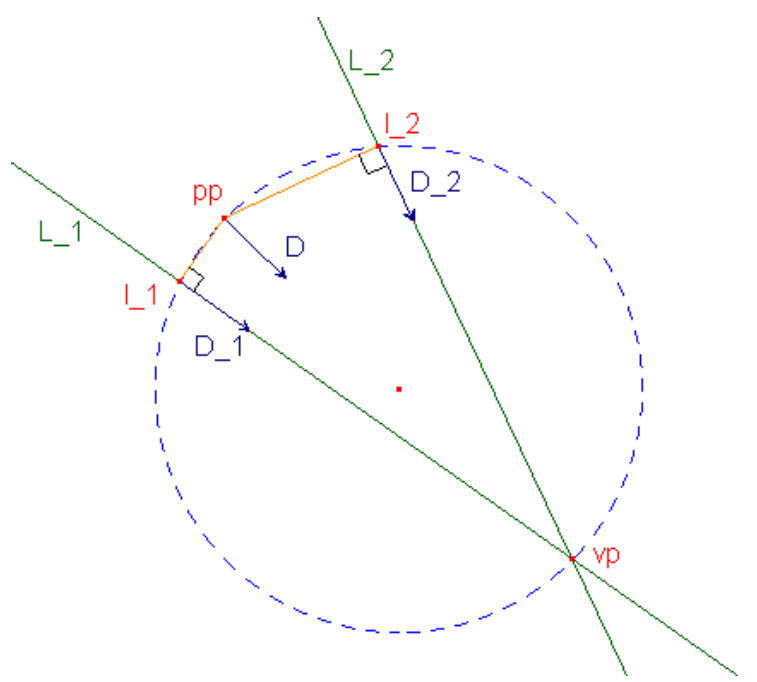

Figure 4: Calculating the vanishing direction $D$ separate from vanishing distance. The fixed point in this parameterization is the principal point $p p . v p$ is the vanishing point of lines $L_{1}$ and $L_{2}$, with $l_{1}$ and $l_{2}$ as their line centers and $D_{1}$ and $D_{2}$ their line directions.

point corresponds with a point at infinity (intersection of parallel lines) and therefore is unaffected by translation. This means that we can calculate the rotation independent from the translation (Caprile and Torre, 1990; Cipolla et al., 1999). We can see this clearly in the projection equation of the vanishing point $V_{i}=\left(u_{i}, v_{i}, w_{i}\right)$, the projection of the point at infinity $D_{i}=\left(x_{D, i}, y_{D, i}, z_{D, i}, 0\right)$ (direction of the parallel lines):

$$
\lambda_{i}\left[\begin{array}{c}
u_{i} \\
v_{i} \\
w_{i}
\end{array}\right]=K[R \mid T]\left[\begin{array}{c}
x_{D, i} \\
y_{D, i} \\
z_{D, i} \\
0
\end{array}\right]
$$

with $\lambda_{i}$ a scale factor, $K$ the calibration matrix with the intrinsic parameters, $R$ the rotation matrix and $T$ the translation vector. Which gives us:

$$
\lambda_{i} K^{-1} V_{i}=R D_{i}
$$

The only unknown factor $\lambda_{i}$ can be calculated using the fact that the inverse of a rotation matrix is its transpose (so $R^{T} R=I$ ) (Foley et al., 1996) and $D_{i}$ is a normalized direction vector (so $D_{i}^{T} D_{i}=1$ ):

$$
\lambda_{i}= \pm \frac{1}{\left|K^{-1} V_{i}\right|}
$$

By using the coordinates of the found vanishing points with their corresponding directions $(\mathrm{X}-, \mathrm{Y}-$ ,Z-axis), the rotation matrix can be calculated from Equation 5. Because of the uncertainties of the sign of $\lambda_{i}$, the orientation is ambiguous but this can be solved by looking at previous frames or adding an extra marker.

The calculation of the 9 unknowns of the $3 \times 3$ rotation matrix seems to require 3 vanishing points. But knowing that the 3 rows of the matrix form an orthogonal base (Foley et al., 1996), we only need 2 correspondences and therefore only 2 axis must be visible at all times (in our case the $\mathrm{X}$ - and Y-axis, the ceiling).

\section{CALCULATING POSITION WITH KNOWN ORIENTATION}

Given the rotation matrix and point or line correspondences between frames, the direction of the translation can be recovered (Caprile and Torre, 1990). The length of the translation is impossible to determine without a reference distance in the input image. In our system we choose to use the known interdistance between LED ropes.

Caprile (Caprile and Torre, 1990) demonstrates that by using 2 image points (with camera directions $\vec{D}_{1}$ and $\overrightarrow{D_{2}}$ ) and known length $\gamma$ and orientation $\vec{D}$ between the world coordinates of points $P_{1}$ and $P_{2}$, we can calculate the distances to both points $(\alpha$ and $\beta$ ) by triangulation (see Figure 5). Thus we have the following system of linear equations with unknowns $\alpha$ and $\beta$ :

$$
\gamma \vec{D}=\beta \vec{D}_{2}-\alpha \overrightarrow{D_{1}}
$$

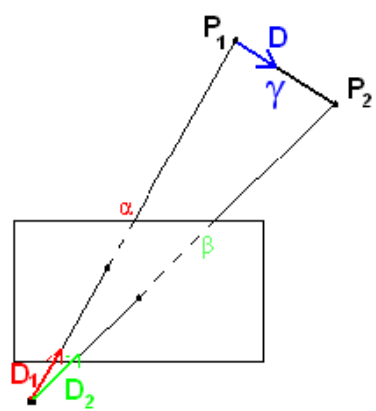

Figure 5: Depth estimation $(\alpha, \beta)$ of two points $P_{1}$ and $P_{2}$ can be done by a simple triangulation given the distance $\gamma$ and spatial orientation $\vec{D}$ of the chosen points.

Given the rotation matrix calculated in the previous step, we undo the rotation on the lines. What we get is a regular grid parallel to the image plane. Since we know the distance between two neighboring lines, the absolute distance to the ceiling can be calculated 
by using triangulation (Equation 7). It is also not required that the $\mathrm{X}$ - and Y-lines are at the same height, if the distance between them is known. This fact makes the construction of our lab set-up easier.

The only remaining unknown is the translation in $\mathrm{X}$ - and $\mathrm{Y}$ - direction. Given the lines in the previous frame, this translation can be trivially calculated.

\section{RESULTS}

The proposed algorithms have been implemented and tested in a virtual set-up as well as in a real room-sized lab set-up.

\subsection{Virtual Set-up}

To evaluate the soundness of our algorithm and design, we first constructed a virtual scene (see Figure 6 ). The virtual scene consists of a 8 by 10 meters room and about 2.5 meters in height. The ceiling of this room consists of a 2D grid of markers (LEDs) in 2 colors: red LEDs to indicate the lines parallel to the $\mathrm{X}$-axis and green to indicate those parallel to the Zaxis. The distance between 2 adjacent parallel lines is known, namely $50 \mathrm{~cm}$.

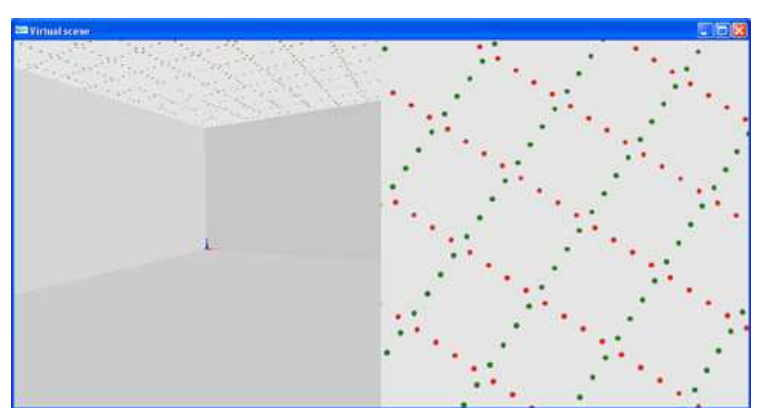

Figure 6: Implementation of the virtual set-up. Left: viewpoint of the user. Right: viewpoint of the tracker camera and input of the tracking system.

A virtual test set-up has the advantage of knowing the exact position and orientation of the user. We use this data to evaluate our tracking system. The test consists of a path through the virtual environment while looking around. The run has been performed on a standard pc and consists of about 400 frames at a resolution of $1024 \times 768$.

Figure 7(a) shows the calculated look- and upvectors defining the orientation of the camera poses of the run. Because we have the real orientation of the camera, we can calculate the absolute RMS error. We find that the accuracy of our algorithm on relatively noise free data is about $0.02^{\circ}$ for Yaw and $0.035^{\circ}$ for
Pitch and Roll.

Using this rotation, the path traveled by the camera can be calculated (Figure 7(b)). We compare the calculated positions with the real poses and get a RMS accuracy of 4 millimeter in X- and Y-directions and 2.4 millimeter accuracy of the height of the camera.

Both orientation and position give a good result under near optimal conditions. Therefore we built a real set-up to test this under real-world conditions.

\subsection{Lab Set-up}

A virtual set-up is good to do the initial testing, but our goal is off course to build a real tracking system. So we constructed a room-sized ( $4 \times 3$ meter) lab set-up as visible on Figure 1. Figure 9 shows a input image of the camera that we want to track. We use a 'Point Grey Flea Firewire' camera capturing $1024 \times 768$ images at $30 \mathrm{fps}$ with a wide field of view camera. Because we use LEDs, the shutter time can be set at as little as $1 \mathrm{~ms}$, which practically eliminates motion blur. This also implies that the system can work in a large variety of lighting conditions, as long as the LED's are the brightest colored features. Figure 8 shows the recording of estimated orientation and position during a walk through our lab set-up.

Unlike the virtual set-up, we cannot compare the results with absolute data. Therefore we captured around 2000 frames at a stationary pose and took the average as the ground truth position. The RMS error of the orientation tracker gives us an accuracy of $0.16^{\circ}$ Yaw and $0.23^{\circ}$ Pitch and Roll. The position data gives us an accuracy of 5 millimeter in $X$ - and Y-direction and 8 millimeter in the Z-direction. If we look at the processor time the camera tracker requires, we see that it does not need more than 11 milliseconds to compute. Most of this computing power (around $9.5 \mathrm{~ms}$ ) is required to segment the LEDs, but still a lot of processor power is left for other tasks or improvements to our algorithm.

During tests, we've seen that the system functions well under varying lighting conditions. However when used with the room lights, the LEDs under the light can't be segmented due to the camera's low dynamic range.

Although the tracking algorithm does not experience any drift, the global pose of persons using the system can differ. Some sort of global starting position must be defined if all participants need to be in the same world space. There is also an inherit ambiguity in the global pose if the user moves when the sensor is occluded. This problem could be improved if we use an inertial sensor when line-of-sight is broken or by placing global positioning beacons. 

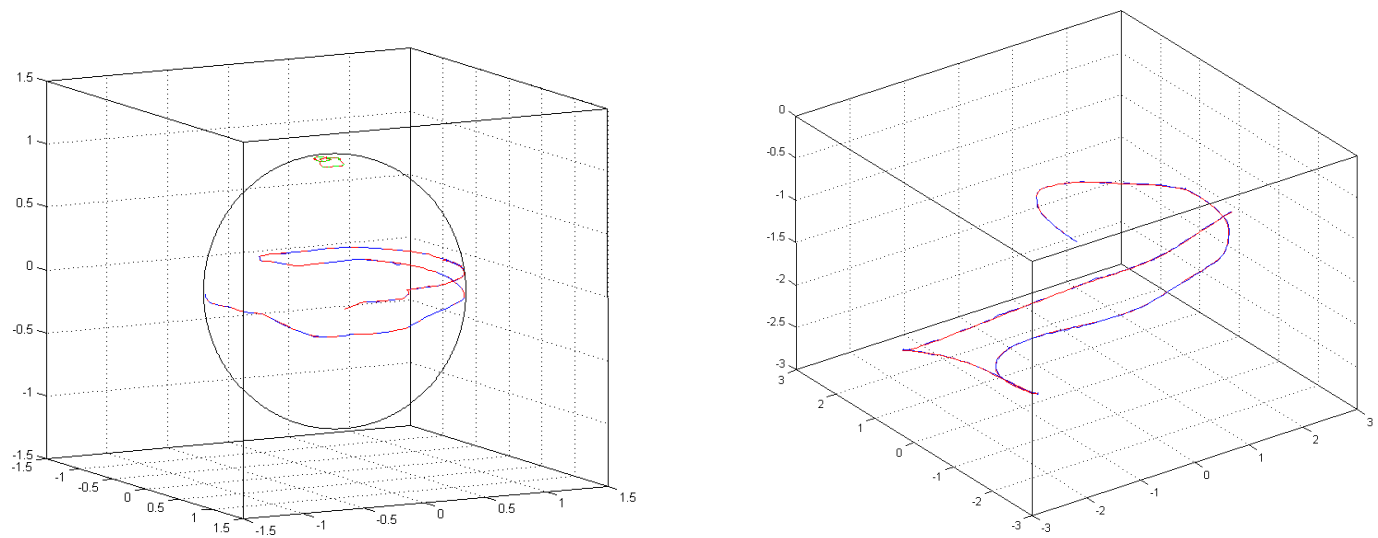

Figure 7: Results of the orientation and position tracker on input of a virtual camera. Left (a): Position of the look vector (green) and up vector (blue) on the unit sphere with the original orientation overlaid (red). Right (b): Position of the camera (blue) with the original position overlaid (red).
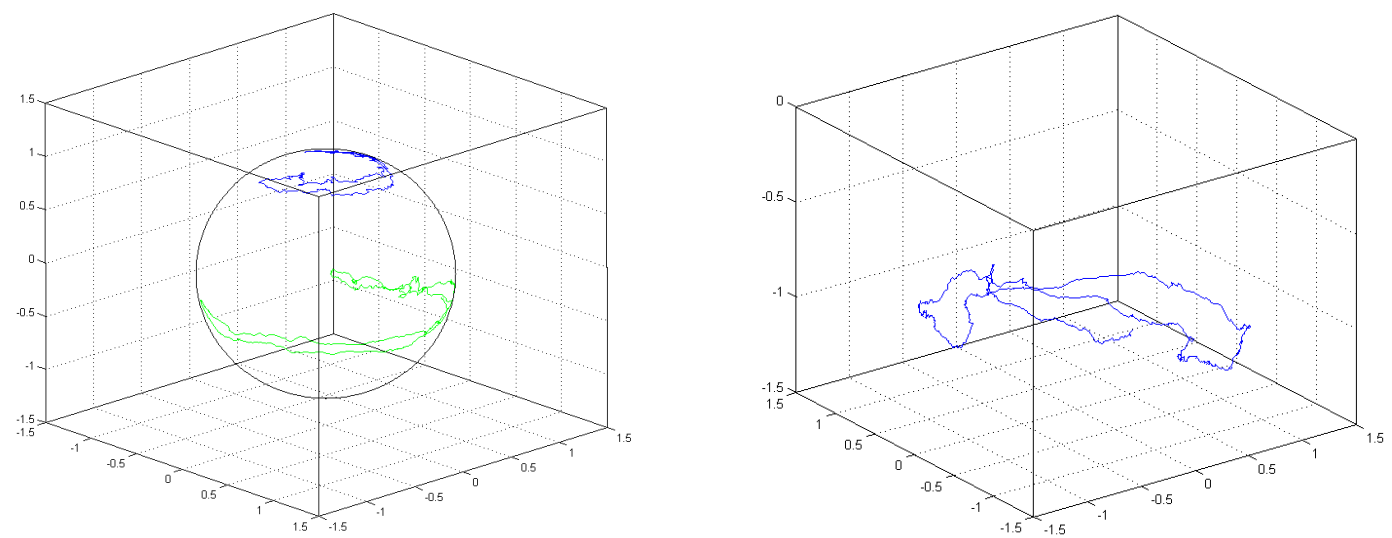

Figure 8: Tracking results of the camera tracker in a room-sized lab set-up. Left (a): Orientation of the calculated camera with the look vector (blue) and up vector (green) on the unit sphere. Right (b): Estimated position of the camera in the lab.

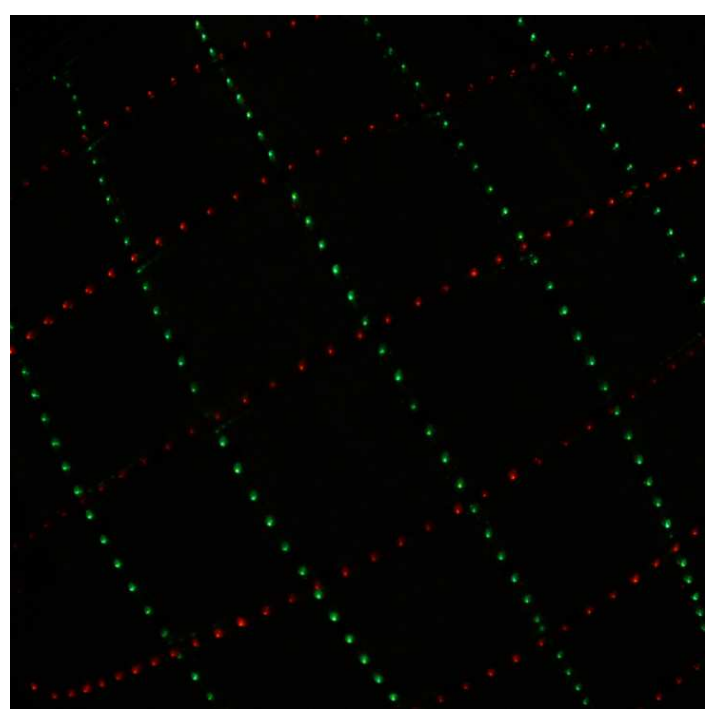

Figure 9: Image from the camera in our lab set-up.

\section{CONCLUSIONS AND FUTURE WORK}

In this paper we have proposed our low-cost widearea optical tracking system using regular cameras and LED ropes. Our proposed real-time orientation tracking algorithm using vanishing points has been shown to be accurate and fast. This could be accomplished using a new parameterization of the Hough transform for detecting line patterns. We are currently looking at iterative refinement algorithms to get even better results.

We are also looking to expand our current test setup to a larger theater room. This may require the placement of global beacons to get an absolute position when line-of-sight is restored.

The first results from our tracking system are very promising for a build-it-yourself wide-area tracker. With orientation accuracy under 0.25 degrees and 
position errors smaller than $1 \mathrm{~cm}$, our system can compete with many expensive commercially available tracking systems on the market.

\section{ACKNOWLEDGEMENTS}

Part of the research at EDM is funded by the ERDF (European Regional Development Fund) and the Flemish government. Furthermore we would like to thank our colleagues for their help and inspiration.

\section{REFERENCES}

Allen, B., Bishop, G., and Welch, G. (2001). Tracking: Beyond 15 minutes of thought. In SIGGRAPH 2001, Course 11 .

Bishop, T. G. (1984). Self-tracker: a smart optical sensor on silicon. $\mathrm{PhD}$ thesis, Chapel Hill, NC, USA.

Bradski, G. (2000). The OpenCV Library. In Dr. Dobbs Journal of Software Tools.

Caprile, B. and Torre, V. (1990). Using vanishing points for camera calibration. In Int. Journal Computer Vision. Kluwer Academic Publishers.

Cipolla, R., Drummond, T., and Robertson, D. (1999). Camera calibration from vanishing points in images of architectural scenes. In BMVC'99, British Machine Vision Conference.

Foley, J. D., van Dam, A., Feiner, S. K., and Hughes, J. F. (1996). Computer graphics: principles and practice (2nd ed. in C). Addison-Wesley Longman Publishing Co.

Foxlin, E. and Naimark, L. (2003). Vis-tracker: a wearable vision-inertial self-tracker. In IEEE Virtual Reality 2003.

Gonzalez, R. C. and Woods, R. E. (2001). Digital Image Processing. Addison-Wesley Longman Publishing Co., New Jersey, 2nd edition.

Hough, P. V. C. (1962). Method and means for recognizing complex patterns. United States Patent 3069654.

Raskar, R., Nii, H., Dedecker, B., Hashimoto, Y., Summet, J., Moore, D., Zhao, Y., Westhues, J., Dietz, P., Barnwell, J., Nayar, S., Inami, M., Bekaert, P., Noland, M., Branzoi, V., and Bruns, E. (2007). Prakash: lighting aware motion capture using photosensing markers and multiplexed illuminators. In SIGGRAPH '07: ACM SIGGRAPH 2007 papers, New York, NY, USA. ACM Press.

Sutherland, I. E. (1968). A head-mounted three dimensional display. In Proceedings of the 1968 Fall Joint Computer Conference, AFIPS Conference Proceedings.

V.Vezhnevets and A.Velizhev (2005). Gml c++ camera calibration toolbox. http://research.graphicon.ru/calibration/gml-c++camera-calibration-toolbox.html.
Wang, J., Azuma, R., Bishop, G., Chi, V., Eyles, J., and Fuchs, H. (1990). Tracking a head-mounted display in a room-sized environment with head-mounted cameras. In Proc. of Helmet-Mounted Displays II.

Ward, M., Azuma, R., Bennett, R., Gottschalk, S., and Fuchs, H. (1992). A demonstrated optical tracker with scalable work area for head-mounted display systems. In SI3D '92: Proceedings of the 1992 symposium on Interactive $3 D$ graphics. ACM Press.

Welch, G., Bishop, G., Vicci, L., Brumback, S., Keller, K., and Colucci, D. (2001). Highperformance wide-area optical tracking: The hiball tracking system. In Presence: Teleoperators and Virtual Environments.

Welch, G. F. (1997). SCAAT: incremental tracking with incomplete information. $\mathrm{PhD}$ thesis, Chapel Hill, $\mathrm{NC}$, USA.

Wormell, D., Foxlin, E., and Katzman, P. (2007). Advanced inertial-optical tracking system for wide area mixed and augmented reality systems. In EGVE 2007, 13th Eurographics Workshop on Virtual Environments. 